\title{
Clinical application value of 3.0T MR diffusion tensor imaging in grade diagnosis of gliomas
}

\author{
WEI SHAN and XING-LONG WANG \\ Medical Image Center, The First People's Hospital of Shangqiu, Shangqiu, Henan 476100, P.R. China
}

Received August 17, 2016; Accepted March 17, 2017

DOI: $10.3892 / 01.2017 .6378$

\begin{abstract}
The clinical value of MR diffusion tensor imaging (DTI) in grade diagnosis of gliomas was investigated. A total of 31 patients with glioma were administered 3.0T MR convention and DTI examination, with quantitative measurement of anisotropy coefficient fractional anisotropy (FA) and apparent dispersion coefficient (ADC) value, and the comparison of quantitative parameters of glioma between low- and high-grade, which was detected by Mann-Whitney $\mathrm{U}$ test. The receiver operation characteristic (ROC) curve was drawn to take the value of ADC and FA in tumor ROI as a critical point, to calculate the area under the curve and to confirm the diagnosis threshold value and evaluate its diagnostic efficiency. The FA value of 14 low-grade glioma cases was $139.4 \pm 81.3$, with an ADC value of $(1.36 \pm 0.21) \times 10^{-3} \mathrm{~mm}^{2} / \mathrm{sec}$. The FA value of 17 high-grade glioma cases was $103.1 \pm 41.5$, with ADC value of $(1.09 \pm 0.28)^{-3} \mathrm{~mm}^{2} / \mathrm{sec}$; the difference between the two groups was statistically significant $(\mathrm{P}<0.05)$. The ADC value was taken as the critical point to judge tumor grade and draw the ROC curve; the area under the curve was 0.79 . As the diagnosis threshold value, the ADC value of $1.11 \times 10^{-3} \mathrm{~mm}^{2} / \mathrm{sec}$ was used to distinguish between low- and high-grade tumor with a sensitivity of $58.8 \%$ and specificity of $92.9 \%$. The FA value was taken as a critical point to judge tumor grade and draw the ROC curve; the area under the curve was 0.62. As the diagnosis threshold value, the FA value of 178.9 was applied to distinguish between low- and high-grade tumor sensitivity of $94.1 \%$ and specificity of $35.7 \%$. Therefore, the FA value and ADC value in DTI has an important estimated value for the pathological grade of glioma.
\end{abstract}

Correspondence to: Dr Wei Shan, Medical Image Center, The First People's Hospital of Shangqiu, 292 Kaixuan South Road, Shangqiu, Henan 476100, P.R. China

E-mail: yxn2361316@163.com

Key words: glioma, MR dynamic contrast-enhanced scan, diffusion tensor imaging, pathology

\section{Introduction}

Glioma is one of the most common primary central nervous system malignant tumors. With relatively high mortality and disability rate, glioma can seriously influence the living quality of patients and cause them and their families heavy economic burden $(1,2)$. Glioma patients account for $\sim 50 \%$ of intracranial tumor patients. Its pathogenesis involves various factors, including genetics, ionizing radiation, biochemical circumstance and environmental pollution and infection. But its exact pathogenetic molecular mechanism remains unclear $(3,4)$. Clinically, glioma is usually diagnosed at the middle and advanced stages with poor prognosis. Therefore, the exploration of imaging features of glioma is of important significance for indications of tumor occurrence, development and prediction of early prognosis.

Diffusion tensor imaging (DTI) mainly reflects the corrosion and damage situation of white matter fiber bundles caused by glioma, and its grade malignancy is associated with the proliferation of tumor cells, and tumor angiogenesis to damage the completeness and continuity of a fiber bundle. The present study quantitatively measured the DTI index of 31 cases with glioma and discuss the clinical application value of DTI technology in establishing glioma grade.

\section{Materials and methods}

Sample selection. The DTI data of 31 patients that have a confirmed diagnosis of glioma by operation and pathology from June, 2013 to January, 2015 was analyzed retrospectively. The cohort included 15 males and 16 females aged 4-70 years and a median age of 43 years. According to Classification and Grading Standard of WHO Central Nervous System Tumors (2007) (2), there are 14 cases of grade I and II glioma, namely low-grade glioma (LGG) and there are 17 cases of grade III and IV, which belong to high-grade glioma (HGG), among which there were 2 cases of pilocytic astrocytoma (grade I), 3 cases of protoplasmic astrocytoma (grade II), 4 cases of diffuse astrocytoma (grade II), 5 cases of oligoastrocytomas (grade II), 9 cases of anaplastic astrocytoma (grade III), 3 cases of anaplastic oligoastrocytomas (grade III), 3 cases of anaplastic ependymoma (grade III) and 2 cases of glioblastoma (grade IV). The main clinical symptoms include headache, dizziness, nausea, sickness, convulsion, acroagnosis and movement 
Table I. General clinical situations of patients.

\begin{tabular}{lcll}
\hline Grade of glioma & Total case no. & Detailed types of glioma & Case no. \\
\hline Low & 31 & Pilocytic astrocytoma(grade I) & $2(2 / 31)$ \\
& 31 & Protoplasmic astrocytoma (grade II) & $3(3 / 31)$ \\
& 31 & Diffuse astrocytoma (grade II) & $4(4 / 31)$ \\
High & 31 & Oligoastrocytomas (grade II) & $5(5 / 31)$ \\
& 31 & Anaplastic astrocytoma (grade III) & $9(9 / 31)$ \\
& 31 & Anaplastic oligoastrocytomas (grade III) & $3(3 / 31)$ \\
& 31 & Anaplastic ependymoma (grade III) & $3(3 / 31)$ \\
& 31 & Glioblastoma (grade IV) & $2(2 / 31)$ \\
\hline
\end{tabular}
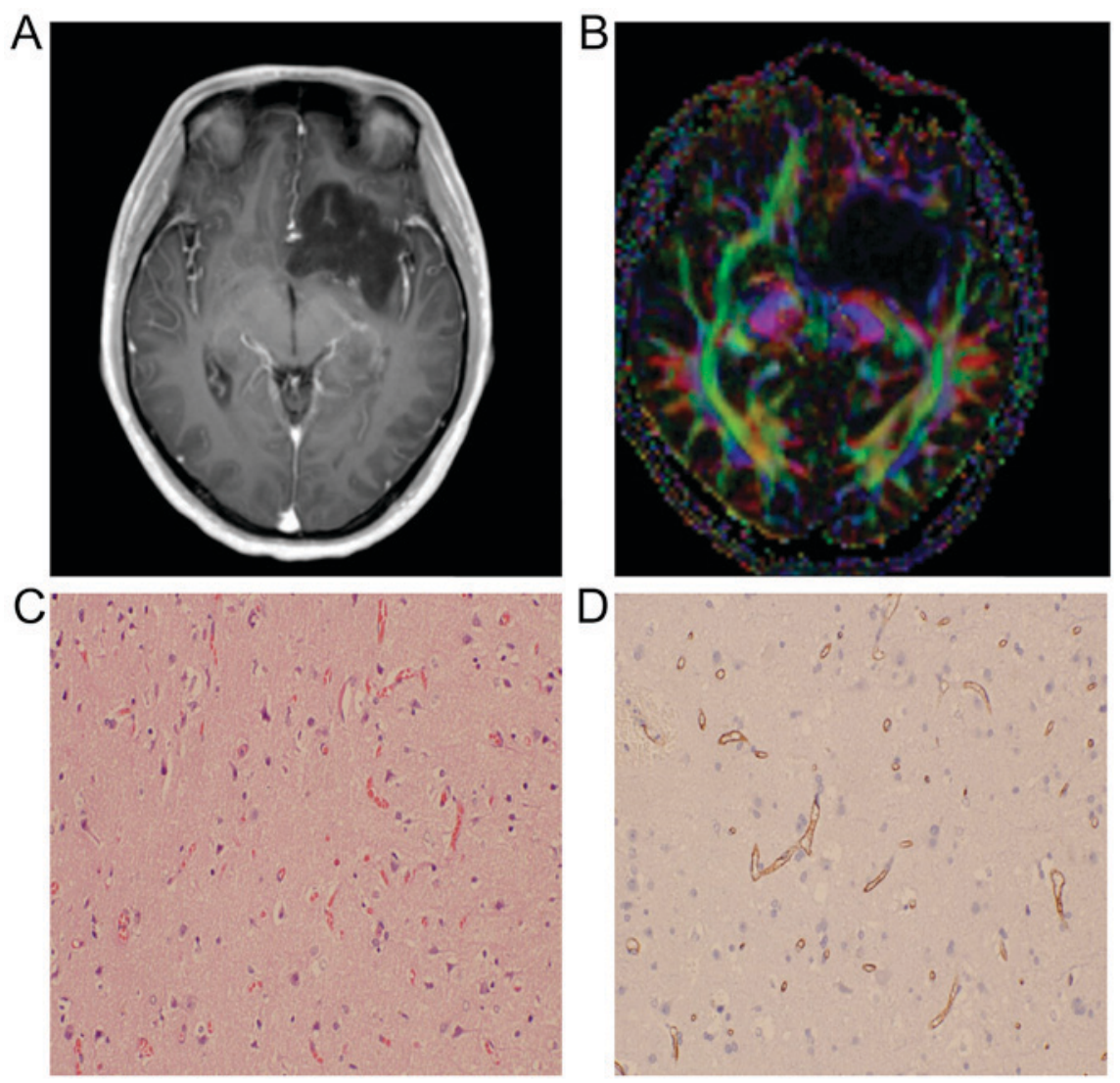

Figure 1. Male, 59 years old, left frontal lobe astrocytoma (WHO grade II). (A) Enhancement scan of left frontal lobe focus, without clear reinforcement; (B) Directionally encoded color image showed low signal of focus and shifted alba under oppression; (C) pathological examination displayed that tumor consisted of neoplastic astrocyte with better differentiation, with loose cell arrangement and mild atypia; (D) CD34 immunohistochemistry stain showing micrangium dispersion, with regular tubular.

disturbance. The general clinical situations of the patient in the two groups is shown in Table I. Images of two cases with astrocytoma or spongioblastoma are shown in Figs. 1 and 2.

MR device and scan scheme. Skyra 3.0T superconductivity MR scanner was obtained from Siemens (Munich, Germany), with scanning sequence including conventional plain scan T1WI and T2WI, fluid-attenuated inversion-recovery sequence (FLAIR), DTI and conventional enhanced MRI scan.

The DTI sequence is as follows: Single-shot echo planner imaging was applied, imaging FOV taking $220 \times 220 \mathrm{~cm}$, with TR time of 5,400 msec, a total of 40 layers, with layer thickness of $3.0 \mathrm{~mm}$, and interlamellar spacing of $0 \mathrm{~mm}$. The phase encoding direction was taken from front to back and a collection technique GRAPPA was run to shorten the time of collecting signal, with 2 accelerated factors and resolution ratio of $128 \times 128$. Multi-direction diffusion imaging scan was applied to exert diffusion sensitive gradient in 30 directions, a $2 \mathrm{~b}$ value taken as 0 and $1,000 \mathrm{sec} / \mathrm{mm}^{2}$, respectively. The average time of collecting signal was 12 times for $b$ value of $0 \mathrm{sec} / \mathrm{mm}^{2}$ and the average time of collecting signal was twice for $b$ value of $1,000 \mathrm{sec} / \mathrm{mm}^{2}$. The DWI, apparent dispersion coefficient (ADC), index ADC and fractional anisotropy (FA) 


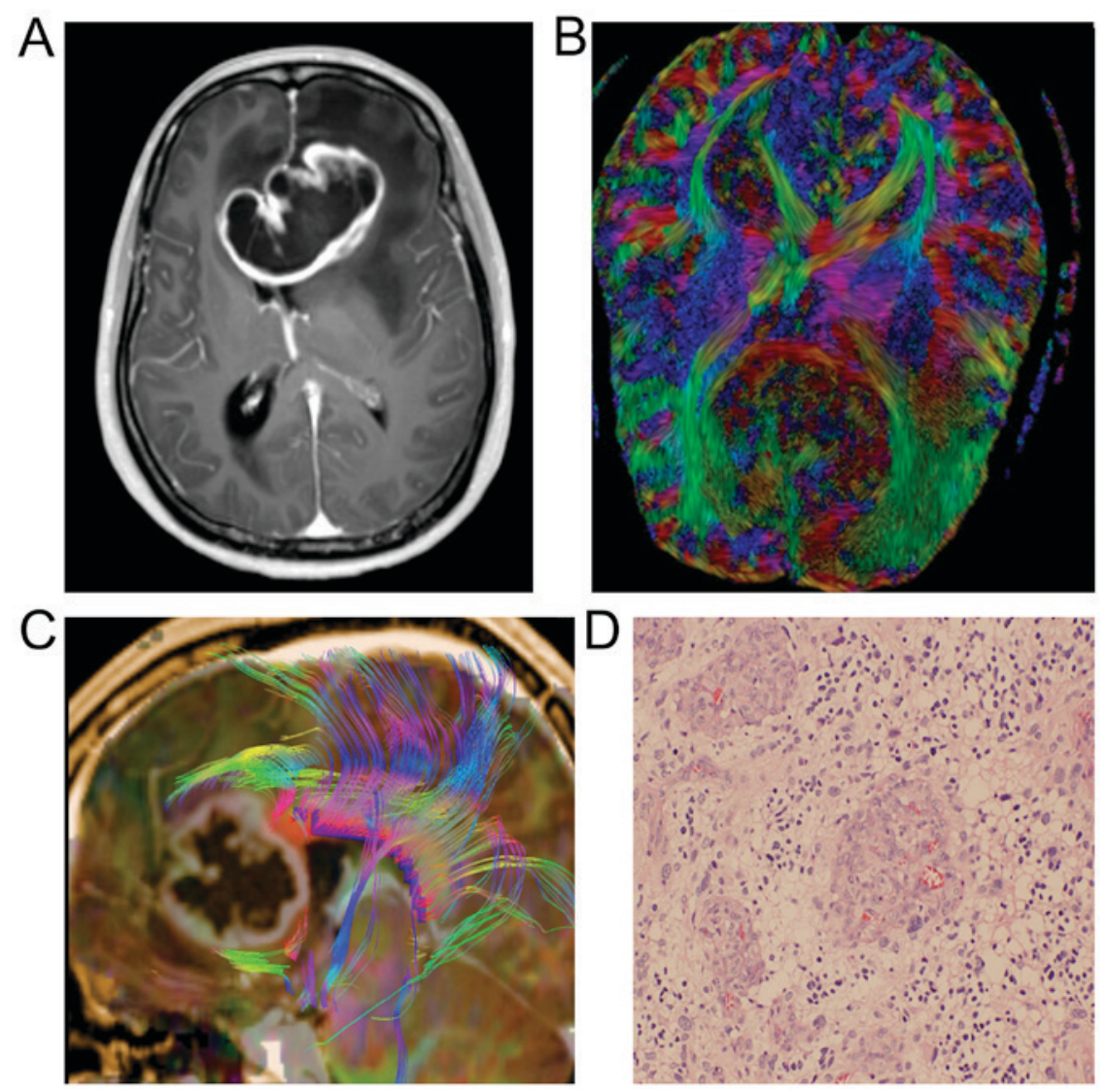

Figure 2. Male, 58 years old, with bilateral frontal lobe spongioblastoma (WHO grade IV). (A) After enhancement, scanning bilateral frontal lobe, with irregular lobulated lesion with obvious wreath reinforcement, uneven wall thickness, internal flakiness necrosis area without reinforcement; (B and C) directionally encoded color and fibet tracking image show focus invasion callosum knee fiber bundle and partial dissolution damage; (D) Pathologic examination displays obvious atypia of focus cell nucleus, cell density increasing significantly, blood vessel distributing as glomerulus.

were processed automatically after scanning and setting in the parameter tab control.

A magnevist solution injection was used as contrastenhancing agent, with a dosage of $0.2 \mathrm{mmol} / \mathrm{kg}$ and injection rate of $2.0 \mathrm{ml} / \mathrm{sec}$. After injection, normal saline of the same dosage was injected. The patients of the present study did not develop an allergic reaction to the contrast agent.

Image processing. The processing and analysis of the DTI image: The original data of DTI from scan was transmitted to Siemens Syngo MMWP workstation and processed by the software package Neuo 3D. Combining directionally encoded color and FA image, 4 ROIs of $30 \mathrm{~mm}^{2}$ were drawn manually on the solid area of the tumor cross section's largest layer. The selection of solid area was referred to as T1, T2, FLAIR and DCE image of three directions, with combination of multiple weighted imaging to observe, judge and avoid edema area, measuring FA value and ADC, and tumor cysts, necrosis and hemorrhage area were avoided when measurement was conducted, the FA value of normal alba taken from the focus as a reference value, and the ratio of lesion and normal alba as the FA value. The ADC value was measured on the ADC image, and 4 ROIs sized $30 \mathrm{~mm}^{2}$ were drawn manually on the solid area of tumor with the largest cross section. The location selection of ROI was brought into correspondence with that of the FA value measurement, taking the average value of the observed value (Fig. 3).
Statistical analysis. The statistical software SPSS 16.0 (SPSS, Inc., Chicago, IL, USA) was used for statistical analysis. The Kolmogorov-Smirnov method was applied to conduct a normality test for the value of FA and ADC, which met normal distribution. The quantitative data are shown as the mean \pm standard deviation and comparison between groups utilized t-test or the $\chi^{2}$ test. The receiver operation characteristic (ROC) curve was used to analyze the FA and ADC value, as well as diagnose the specificity and sensitivity of the threshold value.

\section{Results}

Analysis of FA and ADC value of different pathological patterns of glioma (mean \pm standard deviation). Statistical analysis of the FA and ADC value of 31 glioma cases of different pathological patterns demonstrated that there was no statistical difference among the patients with different pathological patterns $(\mathrm{P}<0.05$; Table II $)$.

Analysis of FA and ADC value of different pathological patterns of glioma. Furthermore, FA and ADC values of different grades of glioma were statistically analyzed, and the results showed that the average FA of LGG was higher than that of HGG group. Moreover, the ADC of LGG was higher than that of HGG group; results were statistically significant (Table III). 
Table II. Analysis of the FA and ADC value of different pathological patterns of glioma (mean \pm standard deviation).

\begin{tabular}{lccc}
\hline Tumor types & Case number & FA value & ADC $\left(10^{-3} \mathrm{~mm}^{2} / \mathrm{sec}\right)$ \\
\hline Pilocytic astrocytoma(grade I) & $2(2 / 31)$ & $106.4 \pm 12.8$ & $1.04 \pm 0.51$ \\
Protoplasmic astrocytoma (grade II) & $3(3 / 31)$ & $113.3 \pm 22.5$ & $1.17 \pm 0.87$ \\
Diffuse astrocytoma (grade II) & $4(4 / 31)$ & $119.2 \pm 18.9$ & $1.21 \pm 0.42$ \\
Oligoastrocytomas (grade II) & $5(5 / 31)$ & $118.7 \pm 21.6$ & $1.09 \pm 0.55$ \\
Anaplastic astrocytoma (grade III) & $9(9 / 31)$ & $121.3 \pm 18.5$ & $1.23 \pm 0.77$ \\
Anaplastic oligoastrocytomas (grade III) & $3(3 / 31)$ & $119.4 \pm 21.7$ & $1.28 \pm 0.69$ \\
Anaplastic ependymoma (grade III) & $3(3 / 31)$ & $125 \pm 18.6$ & $1.32 \pm 0.51$ \\
Glioblastoma (grade IV) & $2(2 / 31)$ & $131.2 \pm 20.7$ & $1.42 \pm 0.87$ \\
Pilocytic astrocytoma(grade I) & - & $>0.05$ & 0.86 \\
Protoplasmic astrocytoma (grade II) & - & & $>0.05$ \\
\hline
\end{tabular}

FA, fractional anisotropy value; ADC, apparent dispersion coefficient value.

Table III. Analysis results of FA and ADC value of different pathological patterns of glioma (mean \pm standard deviation).

\begin{tabular}{lccr}
\hline Tumor grade & Case number & FA value & ADC value $\left(\mathrm{mm}^{2} / \mathrm{sec}\right)$ \\
\hline High-grade glioma & 17 & $103.1 \pm 41.5$ & $(1.09 \pm 0.28) 10^{-3}$ \\
Low-grade glioma & 14 & $139.4 \pm 81.3$ & $(1.36 \pm 0.21) 10^{-3}$ \\
Statistical value & & $\mathrm{t}=-4.610$ & $\mathrm{t}=-3.03$ \\
P-value & & $<0.05$ & $<0.05$ \\
\hline
\end{tabular}

FA, fractional anisotropy value; ADC, apparent dispersion coefficient value.
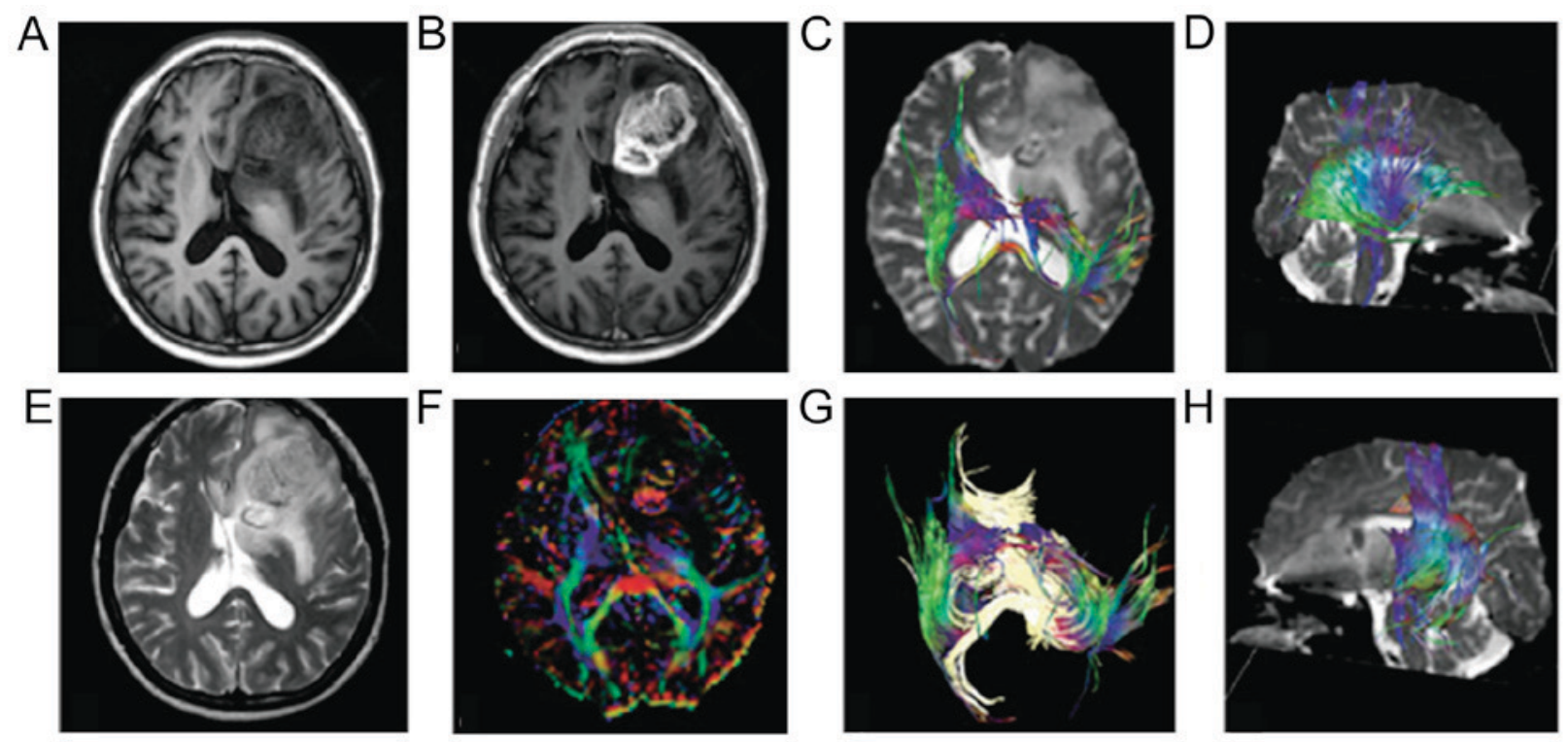

Figure 3. Spongioblastoma, with lesion of left frontal lobe and basal ganglia, T1W1 mixed signal. (A) T2W1 high mixed signal, (B) uneven regiment massive enhancement and (C) peripheral edema. (D) FA image red callosum knee fibers, green left frontal lobe fiber infiltration, under oppression to shift, with (E-H) fiber tractogrpahy display.

Results of the analysis of area under ROC curve. The $\mathrm{ADC} / \mathrm{FA}$ value in tumor ROI was taken as the critical point to judge tumor grade and draw the ROC image (Fig. 4); the area under the curve was $0.79 / 0.62$. As the diagnosis threshold value, the ADC/FA value of $1.11 \times 10^{-3} \mathrm{~mm}^{2} / \mathrm{sec} / 178.9$ was used to distinguish between low- and high-grade tumors. Using this threshold value, high-grade malignant tumors were diagnosed with sensitivity of 58.8/94.1\% and specificity of $92.9 / 35.7 \%$. Compared to FA, ADC has greater advantage in distinguishing tumor grade. 
Table IV. Analysis of sensitivity and specificity of conventional and DTI combination scan.

Pathological results

Groups

High-grade glioma

Low-grade glioma

Total

\section{Conventional MRI}

High-grade glioma

Low-grade glioma

Total

Conventional MRI combined with DTI

High-grade glioma

Total

DTI, diffusion tensor imaging.

Table V.Comparison of accuracy, sensitivity, specificity, positive and negative predictive value of different detection methods (\%).

\begin{tabular}{lcccc}
\hline $\begin{array}{l}\text { Variables } \\
\text { Conventional }\end{array}$ & Sensitivity $(\%)$ & Specificity $(\%)$ & $\begin{array}{c}\text { Positive } \\
\text { predictive value (\%) }\end{array}$ & $\begin{array}{c}\text { Negative } \\
\text { predictive value }(\%)\end{array}$ \\
$\begin{array}{l}\text { MRI } \\
\text { Conventional MRI }\end{array}$ & 76.1 & 86.2 & 81.1 & 0.75 \\
$\begin{array}{l}\text { combined DTI } \\
\text { T-value }\end{array}$ & 100 & 100 & 55.0 & 45.0 \\
P-value & 1.27 & 1.88 & 1.49 & 1.57 \\
\end{tabular}

DTI, diffusion tensor imaging.

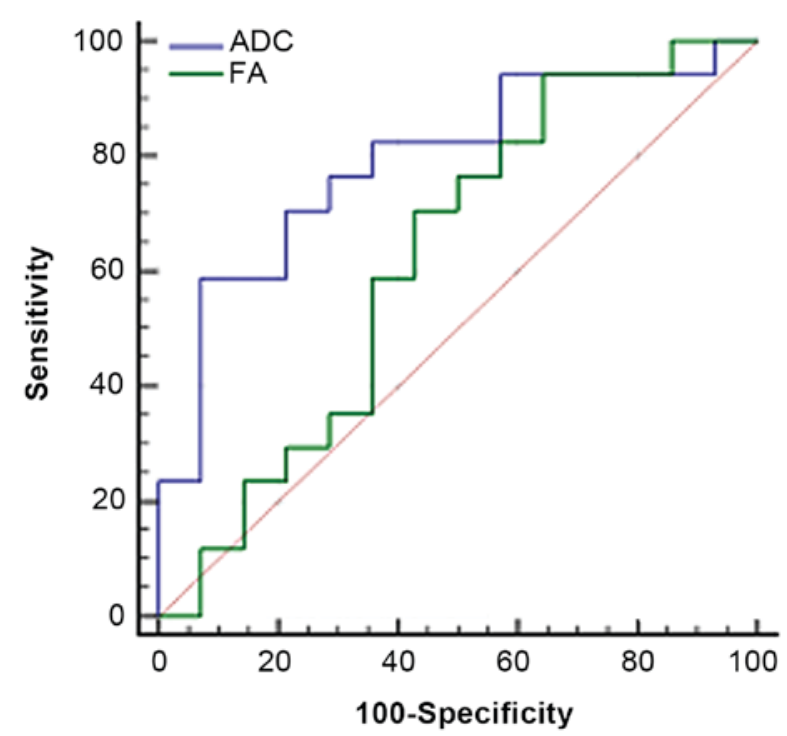

Figure 4. Receiver operation characteristic curve that takes ADC/FA value as critical point to judge low- and high-grade tumor, with area under curve of 0.79 (ADC)/0.62 (FA). ADC, apparent dispersion coefficient, FA, fractional anisotropy.

Analysis of sensitivity and specificity of conventional and DTI combination scan. Analysis of HGG as the positive, sensitive, specific, positive and negative predictive value of conventional MRI and DTI combination scan was conducted. Our analysis demonstrated that the sensitivity, specificity and accuracy of DTI combination scan were higher than that of conventional scans; differences were statistically significant $(\mathrm{P}<0.05$; Tables IV and V).

\section{Discussion}

The guiding significance of the MR DTI index is as follows: The subsidiarity that iconography can provide for tumor grading is correspondingly indispensable due to the importance of tumor grading for treatment and prognosis. DTI mainly reflects the integrity of the nerve fiber anatomical structure which can assess the microcosmic physiology and pathological state of white matter fiber tracts using non-invasive techniques. Moreover, DTI technology can also judge tumor cell density and evaluate the ability of tumor cells to invade white matter degree efficiently.

The change of ADC value in tumors is influenced by tumor cell structure to a great extent (5). With the increasing ascendance of grade malignancy and the density of tumor cell structure, the tumor form becomes more complicated. Brownian movement of molecules is limited even further, eventually leading to decline of the ADC value directly. In 
the present study, the ADC value in HGG is lower than that in LGG, which indicates that tumor cell structures will become more complicated with an increase of grade malignancy, albeit indirectly. In addition, HGG with high-grade malignancy can lead to abnormal tumor blood vessel hyperplasia and the micro-structure completeness of the tumor cell is broken, which results in free water infiltration nerve fiber axon gap and a decline in the FA value. Through the comparison of FA values, the present study found that when compared to LGGs, the FA value of HGG is reduced significantly, which reflects blood vessels in HGG mesenchyme erodes or damage to nerve fiber bundle. This leads to free water infiltration to nerve fiber axon gap and decline in the FA value, which suggests that it indirectly leads to the spatial distribution situation of tumor capillaries (5-7).

Previous research results indicate that the difference between FA and ADC in high- and low-grade glioma was statistically significant; our study corroborated the results that both of them can distinguish different grades of the tumor (4). However, in terms of the comparison of diagnostic efficiency, the FA value of different grades as the critical point to distinguish tumor grade had a curve area of 0.62 , which was lower than that of ADC; this suggests that ADC has a better diagnostic efficiency. However, FA has a stronger sensitivity and a lower specificity and ADC has lower sensitivity and stronger specificity; the difference is attributed to the individual theory behind these two parameters (8). FA mainly reflects anisotropy of the internal structure of tumor, while ADC reflects a limited degree of molecular diffusion into the tumor, which is a comprehensively average effect, excluding anisotropy (9-11). Tumors are heterogeneous, and therefore, there is coexistence of edema and substance as well as coexistence of low- and high-grade. If we evaluated the FA and ADC from two different starting points, there would be a different analysis result (12-14).

The present study utilized the FA and ADC to evaluate high- and low-grade of tumors, revealing that the two indexes can distinguish between the grades of tumor. The combination of these is more helpful in distinguishing the grade of tumor and understanding the internal structure, which can also be applied for a study with a larger sample size. We analyzed the sensitivity, specificity, accuracy, positive predictive value and negative predictive value of conventional MRI and DTI combination and found that the sensitivity, specificity and accuracy of DTI combination scan was higher than that of conventional MRI scan; differences were statistically significant $(\mathrm{P}<0.05)$. We speculated that DTI method was superior in the evaluation of blood vessel corrosion and damage to the nerve fiber bundle, which results in free water infiltration to nerve fiber axon gap. Therefore, it has a favorable reminder for clinical diagnosis, which is helpful for a pathologist to diagnose the glioma grade.
A serious limitation of this study is that there was a limited number of clinical cases, and therefore, there was a possible existence of a bias in the evaluation of diagnostic efficiency. In conclusion, conventional and DTI combination scan can assess glioma with new vessel hyperplasia and the degree of nerve fiber bundle damage more comprehensively, which can provide a new method for the monitoring of tumor diagnosis, growth, transfer and postoperative recurrence.

\section{References}

1. Inoue T, Ogasawara K, Beppu T, Ogawa A and Kabasawa H: Diffusion tensor imaging for preoperative evaluation of tumor grade in gliomas. Clin Neurol Neurosurg 107: 174-180, 2005.

2. Rousseau A, Mokhtari K and Duyckaerts C: The 2007 WHO classification of tumors of the central nervous system - what has changed? Curr Opin Neurol 21: 720-727, 2008.

3. Field AS and Alexander AL: Diffusion tensor imaging in cerebral tumor diagnosis and therapy. Top Magn Reson Imaging 15: $315-324,2004$

4. Nimsky C, Ganslandt O, Hastreiter P, Wang R, Benner T, Sorensen AG and Fahlbusch R: Preoperative and intraoperative diffusion tensor imaging-based fiber tracking in glioma surgery. Neurosurgery 61: 178-185, discussion 186, 2007.

5. Hadziahmetovic M, Shirai K and Chakravarti A: Recent advancements in multimodality treatment of gliomas. Future Oncol 7: 1169-1183, 2011.

6. Yao K, Wang H, Duan Z, Bian Y, Xia L, Ma Z and Qi X: Mixed granular cell astrocytoma and fibrosarcoma of the brain: A case report. Int J Clin Exp Pathol 7: 4473-4478, 2014.

7. Leestma JE: Brain tumors. Am J Pathol 100: 239-316, 1980

8. Wang H, Li XT, Wu C, Wu ZW, Li YY, Yang TQ, Chen GL, Xie XS, Huang YL, Du ZW, et al: miR-132 can inhibit glioma cells invasion and migration by target MMP16 in vitro. Onco Targets Ther 8: 3211-3218, 2015.

9. Doblas S, He T, Saunders D, Pearson J, Hoyle J, Smith N, Lerner M and Towner RA: Glioma morphology and tumor-induced vascular alterations revealed in seven rodent glioma models by in vivo magnetic resonance imaging and angiography. J Magn Reson Imaging 32: 267-275, 2010.

10. Bokacheva L, Ackerstaff E, LeKaye HC, Zakian K and Koutcher JA: High-field small animal magnetic resonance oncology studies. Phys Med Biol 59: R65-R127, 2014.

11. Lohrke J, Frenzel T, Endrikat J, Alves FC, Grist TM, Law M, Lee JM, Leiner T, Li KC, Nikolaou K, et al: 25 years of Contrast-Enhanced MRI: Developments, current challenges and future perspectives. Adv Ther 33: 1-28, 2016.

12. Gutova M, Frank JA, D'Apuzzo M, Khankaldyyan V, Gilchrist MM, Annala AJ, Metz MZ, Abramyants Y, Herrmann KA, Ghoda LY, et al: Magnetic resonance imaging tracking of ferumoxy tol-labeled human neural stem cells: Studies leading to clinical use. Stem Cells Transl Med 2: 766-775, 2013.

13. Kalpathy-Cramer J, Gerstner ER, Emblem KE, Andronesi OC and Rosen B: Advanced magnetic resonance imaging of the physical processes in human glioblastoma. Cancer Res 74: 4622-4637, 2014

14. Zhou J, Tryggestad E, Wen Z, Lal B, Zhou T, Grossman R, Wang S, Yan K, Fu DX, Ford E, et al: Differentiation between glioma and radiation necrosis using molecular magnetic resonance imaging of endogenous proteins and peptides. Nat Med 17: 130-134, 2011. 